\title{
Residence Time Statistics for Normal and Fractional Diffusion in a Force Field
}

\author{
E. Barkai ${ }^{1}$ \\ ${ }^{1}$ Department of Physics, Bar Ilan University, Ramat-Gan 52900 Israe
}

\begin{abstract}
We investigate statistics of occupation times for an over-damped Brownian particle in an external force field. A backward Fokker-Planck equation introduced by Majumdar and Comtet describing the distribution of occupation times is solved. The solution gives a general relation between occupation time statistics and probability currents which are found from solutions of the corresponding problem of first passage time. This general relationship between occupation times and first passage times, is valid for normal Markovian diffusion and for non-Markovian sub-diffusion, the latter modeled using the fractional Fokker-Planck equation. For binding potential fields we find in the long time limit ergodic behavior for normal diffusion, while for the fractional framework weak ergodicity breaking is found, in agreement with previous results of Bel and Barkai on the continuous time random walk on a lattice. For non-binding potential rich physical behaviors are obtained, and classification of occupation time statistics is made possible according to whether or not the underlying random walk is recurrent and the averaged first return time to the origin is finite. Our work establishes a link between fractional calculus and ergodicity breaking.
\end{abstract}

PACS numbers:

\section{INTRODUCTION}

Consider the trajectory of a single Brownian particle. The total time the particle spends in a given domain is called the residence time or the occupation time. The well known example is P. Lévy's arcsine law [1, 2]. Consider a Brownian motion $\dot{x}(t)=\eta(t)$ where $\eta(t)$ is Gaussian white noise with zero mean. Lévy investigated the residence time of the particle in the domain $x>0$, which we call $T^{+}$, when the motion is unbounded and the total observation time is $t$. Naive expectation is that $T^{+} / t=1 / 2$ with small fluctuations when $t \rightarrow \infty$, namely the particle occupies the domain $x>0$ for half of the time of observation. However, instead the probability density function of $T^{+} / t$ is given by the well known arcsine law, $f\left(T^{+} / t\right)=\left[\pi \sqrt{\left(T^{+} / t\right)\left(1-T^{+} / t\right)}\right]^{-1}$ with $0 \leq T^{+} / t \leq 1$. This probability density has a U shape, which means that for a typical realization of the Brownian trajectory, the particle spends most of the time in one half of space (say $x>0)$ and not in the other $(x<0)$.

Many extensions of this well known result are found in the literature. Darling and Kac [3, 44 found the limiting distribution of the time spent in a domain in two dimensions, and this line of investigation was extended to three dimension by Berezhkovskii et al [5] . Lamperti's [6] limit theorem gives a very general mathematical foundation for occupation time statistics (see more details in the manuscript). Recently in 7] Pearson's type of ballistic motion with random reorientation was considered, instead of the usual assumption of an underlying continuum process. The basic mathematical theory for the calculation of occupation time statistics for Brownian motion was developed by Kac, and is usually based on the Feynmann-Kac formula (see [1, 8] and Ref. therein).

*Electronic address: barkaie@mail.biu.ac.il
Statistics of occupation times is of-course not limited to Brownian motion and diffusion, and it is a topic of wide investigation [1], for example in the context of renewal processes 9|, theory and experiments of blinking quantum dots [10, 11], weak ergodicity breaking of dynamics generated using deterministic maps 12], and work distribution functions of a single spin 13].

The problem of occupation times of a Brownian particle in the presence of external field was consider recently, by Majumdar and Comtet 14]. Using the Kac formalism [1] they found a backward Fokker-Planck equation whose solution yields statistics of occupation times. In [14] the problem of occupation time statistics of a particle performing a random walk on a random walk i.e. the Sinai model was investigated. It was shown that statistics of occupation times are drastically changed when averages over random disorder are made.

In the first part of this manuscript we consider the problem of occupation times for normal Brownian motion in an external fields. We solve exactly the backward Fokker-Planck equation given in [14]. This solution gives a general relation between occupation time and first passage time statistics. Besides the theoretical interest in such a relation, the solution is used to classify very general behaviors of occupation times based on the corresponding properties of the first passage times. The later are investigated in great detail in the literature [2], and we can use this knowledge to solve analytically the problem of occupation times at-least for some simple cases. For example we show that in the limit of long measurement times, and for binding force fields, statistics of occupation times is determined by Boltzmann's statistics, namely the underlying dynamics is ergodic, as expected.

Statistics of occupation time is important from a fundamental point of view, since if we are able to calculate statistics of occupation times from some underlying dynamics, one can check the validity of the ergodic hypothesis and its possible extensions. A trivial example 
is Gaussian Brownian motion in a system of finite size $0<x<L$, in the absence of external force fields. Then it is easy to show that the residence time in half of the system i.e. in the domain $(0, L / 2)$, is in statistical sense half of the observation time, when the observation time is long, as expected. For dynamics described by fractional kinetic equations 15, 16], we show that such a simple ergodic picture does not hold.

In the second part of the paper we consider the problem of a particle undergoing an anomalous diffusion process. We model this behavior using the fractional time FokkerPlanck equation [17, 18]. This fractional framework is based on fractional calculus e.g. $d^{1 / 2} / d t^{1 / 2}$, which is briefly introduced in the manuscript. We show for example, that the general relation between occupation times and first passage times we find in the first part of the paper is still valid, even for the non-Markovian sub-diffusive case. Similar to normal diffusion case a classification of typical behaviors of occupation times is found, and analytical solutions provided. For dynamics in binding force fields we find weak ergodicity breaking. In the conclusions we compare our results on occupation times found here using the fractional framework, and recent results of Bel and Barkai [19, 20] on statistics of residence times for continuous time random walks.

For applications, residence times are of interest in the context of chemical reactions [21, 22, 23] and rather generally for statistical analysis of experimental data. Residence times are very important in the context of single molecule dynamics 24, 25]. It is now possible to follow dynamics of single molecules embedded in condensed phase environments, using optical techniques. For example dynamics of single molecules in cells or in solution are used to follow chemical reactions in real time, without the problem of ensemble averaging found in usual measurements. A typical experiment uses a laser to investigate the dynamics of a particle. In many cases and under certain conditions 24] if a particle or a reaction coordinate is in a finite domain, the system may emit photons, while when the particle is out of the domain the system does not emit. Very briefly, the domain width can be imagined as the width of the laser beam in single molecule fluorescence experiments when the particle comes in and out of resonance with the exciting laser field, due to its diffusion in space e.g. 26], or it could be the Föster radius in fluorescence resonance energy transfer measurement e.g. 27]. Thus the total time the photons are emitted is approximately the residence time, which is proportional to the number of emitted photons, which is generally a random variable. For other sources of fluctuations in single molecule experiments see [24].

\section{NORMAL DIFFUSION}

Consider a one dimensional over-damped Brownian motion in an external force field $F(x)$. The Smoluchowski Fokker-Planck equation for the concentration of non in- teracting particles is

$$
\frac{\partial c(x, t)}{\partial t}=D\left[\frac{\partial^{2}}{\partial x^{2}}-\frac{\partial}{\partial x} \frac{F(x)}{k_{b} T}\right] c(x, t),
$$

where $T$ is the temperature and $D$ is the diffusion coefficient. As well known the equilibrium of the ensemble of particles is the Boltzmann equilibrium, provided that the force field is binding.

Consider a single particle, which at time $t=0$ is on $x_{0}$, the observation time of the stochastic dynamics is $t$. The random variable we investigate here is $T^{+}$, the total time the particle occupies the region $x>0$. In principle during the observation time the particle may cross the point $x=0$ many times, and then the occupation time $T^{+}$is composed of many sojourn times in $x>0$.

Let $P_{x_{0}, t}\left(T^{+}\right)$be the probability density function (PDF) of $T^{+}$. The double Laplace transform

$$
P_{x_{0}, s}(u)=\int_{0}^{\infty} \int_{0}^{\infty} e^{-s t} e^{-u T^{+}} P_{x_{0}, t}\left(T^{+}\right) \mathrm{d} t \mathrm{~d} T^{+},
$$

is defined so that $s$ and $t$ and $u$ and $T^{+}$are Laplace pairs. Majumdar and Comtet [14] found the equation of motion for $P_{x_{0}, t}\left(T^{+}\right)$in double Laplace space

$D\left[\frac{\partial^{2}}{\partial x_{0}^{2}}+\frac{F\left(x_{0}\right)}{k_{b} T} \frac{\partial}{\partial x_{0}}\right] P_{x_{0}, s}(u)-\left[s+\Theta\left(x_{0}\right) u\right] P_{x_{0}, s}(u)=-1$.

Where $\Theta\left(x_{0}\right)$ is the step function: $\Theta\left(x_{0}\right)=1$ if $x_{0}>0$ otherwise it is zero. This type of equation is called a backward Fokker-Planck equation, the operator on the left hand side depends on the initial condition $x_{0}$. Eq. (3) is solved for the matching boundary conditions

$$
\begin{gathered}
\left.P_{x_{0}, s}(u)\right|_{x_{0}=0^{+}}=\left.P_{x_{0}, s}(u)\right|_{x_{0}=0^{-}}, \\
\left.\frac{\partial P_{x_{0}, s}(u)}{\partial x_{0}}\right|_{x_{0}=0^{+}}=\left.\frac{\partial P_{x_{0}, s}(u)}{\partial x_{0}}\right|_{x_{0}=0^{-}} .
\end{gathered}
$$

We will re-derive Eq. (3) later as a special limiting case of a more general non-Markovian dynamics.

To prepare for the solution of Eq. (3) we define the following survival probabilities. The probability that a particle starting at $x_{0}$ with $x_{0}<0$, to remain in the domain $x<0$ without leaving it even once, during the time $t$ is the survival probability $W_{x_{0}}^{-}(t)$. Let $W_{x_{0}}^{-}(s)$ be the Laplace transform of $W_{x_{0}}^{-}(t)$ and similarly the Laplace transform of the survival probability in the domain $x>0$ is $W_{x_{0}}^{+}(s)$ for $x_{0}>0$. The key to the solution of the problem of occupation times in half space, is to recall Tachiya's equation for the survival probability of a particle in half space [28, 29]

$D\left[\frac{\partial^{2}}{\partial x_{0}^{2}}+\frac{F\left(x_{0}\right)}{k_{B} T} \frac{\partial}{\partial x_{0}}\right] W_{x_{0}}^{-}(s)-s W_{x_{0}}^{-}(s)=-1 \quad x_{0}<0$,

and a similar equation holds for $x_{0}>0$. The boundary conditions for Eq. (5) are the standard conditions 
used for the calculation of survival probabilities. Namely, $\left.W_{x_{0}}^{-}(s)\right|_{x_{0}=0}=0$, means that the particle reaches the boundary on $x=0$ instantaneously if the particle starts very close to the absorbing boundary and if $x_{0} \rightarrow-\infty$ survival is unity.

The solution of Eq. (3) for the occupation time is

$$
P_{x_{0}, s}(u)=W_{x_{0}}^{-}(s)+\left[1-s W_{x_{0}}^{-}(s)\right] G_{s}(u)
$$

if $x_{0}<0$,

$$
P_{x_{0}, s}(u)=W_{x_{0}}^{+}(s+u)+\left[1-(s+u) W_{x_{0}}^{+}(s+u)\right] G_{s}(u)
$$

if $x_{0}>0$. From Eq. (6) the physical meaning of $G_{s}(u)$ becomes clear, it is the double Laplace transform of $G_{t}\left(T^{+}\right)$the PDF of the random variable $T^{+}$ for a particle starting on $x_{0}=0$. The $\mathrm{PDF} G_{t}\left(T^{+}\right)$ contains the information on the problem of occupation times, while the survival probability was investigated previously by many authors, hence in what follows we investigate $G_{t}\left(T^{+}\right)$. Using Eq. (5) the reader can verify that Eq. (6) is indeed the general solution of the problem of occupation times Eq. (3). Using the boundary condition $W_{x_{0}=0}^{-}(s)=W_{x_{0}=0}^{+}(s)=0$ and the solution Eq. (6) it is easy to see that the boundary condition $\left.P_{x_{0}, s}(u)\right|_{x_{0}=0^{+}}=\left.P_{x_{0}, s}(u)\right|_{x_{0}=0^{-}}=G_{s}(u)$ in Eq. (44) is satisfied.

The second matching boundary condition in Eq. (4), on the derivatives of $P_{x_{0}, s}(u)$ yields $G_{s}(u)$ using Eq. (6)

$$
G_{s}(u)=\frac{J^{+}(s+u)-J^{-}(s)}{(s+u) J^{+}(s+u)-s J^{-}(s)}
$$

where the currents are

$J^{+}(s+u)=\left.\frac{\partial W_{x_{0}}^{+}(s+u)}{\partial x_{0}}\right|_{x_{0}=0^{+}}, \quad J^{-}(s)=\left.\frac{\partial W_{x_{0}}^{-}(s)}{\partial x_{0}}\right|_{x_{0}=0^{-}}$.

Eqs. (6 17) are the main results so far since they yield a general relation between statistics of occupation times and survival probability currents. From Eq. (8) we see that the solution of the problem of occupation times is found in terms of two solutions of the corresponding first passage time problems, the first for a particle starting on $x_{0}>0$ and absorbed on $x=0$ (i.e. $J^{+}$) and the second for a particle starting on $x_{0}<0$ and absorbed on $x=0$ (i.e. $J^{-}$). Thus the problem of residence times is solved in three steps:

(i) Find solutions of two first passage time problems for, $x_{0}>0$ and $x_{0}<0$ in Laplace space.

(ii) Use Eq. (7) to find the solution of the problem of residence times in double Laplace space.

(iii) And then use a two dimensional inverse Laplace transform to get $G_{t}\left(T^{+}\right)$from $G_{s}(u)$.

Since there exists a vast literature on the solutions of the problem of first passage time [2], the relationship Eq. (7) is very useful for the calculation of statistics of occupation times. We note that some connections between first passage times and occupation times, which are different and in our opinion less general than Eq. (8), appeared previously in the literature [5, ㅇ, 20]. Finally, while we considered the occupation time in half space, occupation times in a finite domain are also obtained in a similar way, and it is straight forward to extended our results to higher dimensions.

Majumdar and Comtet [14] classify statistics of occupation times according to behavior of the potential field, in particular they consider motion in stable, unstable and flat potential fields. Here the relation between survival currents and statistics of occupations times, Eq. (7) can be used to characterized certain very general and new behaviors of occupation times.

Survival probabilities in a finite and infinite domain exhibit three well known typical physical behaviors [2], we consider the right random walk (i.e. $x_{0}>0$ ) and similar classification holds for the left random walk. Later we will classify behaviors of residence times based on these three behaviors of first passage times.

Case 1 The random walk is recurrent, and the average first passage time from $x_{0}$ to 0 is finite. Such cases correspond to diffusion in a system of finite size, when the particle cannot escape to infinity, e.g. the driving force field is binding.

Case 2 The random walk is transient, i.e. the survival probability in $x>0$ is finite in the limit of long times. Such cases happen when the external force drives the particle far from the origin, and the system is infinite. In that case in the limit of small $s$

$$
W_{x_{0}}^{+} \sim \frac{\epsilon_{x_{0}}^{+}}{s}
$$

where $\epsilon_{x_{0}}^{+}$is the survival probability of the particle starting on $x_{0}$, without reaching $x=0$, when $t \rightarrow \infty$. Similar notation is used for the left random walk, with $\epsilon_{x_{0}}^{-}$.

Case 3 Random walks are recurrent, though the average first passage time is infinite. A particularly common situation is the case when the survival probability decays like $t^{-1 / 2}$ for long times. This happens if the non-diverging external field $F(x)=0$ for $x>x_{c}$ and the system is infinite, namely when diffusion controls the long time dynamics. For such a case [2]

$$
W_{x_{0}}^{+} \sim \frac{A_{x_{0}}^{+}}{s^{1 / 2}}, \quad s \rightarrow 0,
$$

where $A_{x_{0}}^{+}>0$ depends of-course on the details of the force field.

We now consider certain general properties of the statistics of occupation times for the three cases.

Case 1 The long time behavior of $G_{t}\left(T^{+}\right)$is now considered. When the left and right random walks, starting at $x_{0}<0$ or $x_{0}>0$, respectively, are recurrent and the average first passage time is finite. For this case the small $s$ limit yields

$$
W_{x_{0}}^{ \pm}(s=0)=\left\langle t_{x_{0}}^{ \pm}\right\rangle,
$$

where $\left\langle t_{x_{0}}^{ \pm}\right\rangle$is the average time for the particle starting on $x_{0}<0$ (or $x_{0}>0$ ) to reach the origin for the first 
time. The small $s$ and $u$ limit, their ratio arbitrary, of Eq. (7) gives the long $t$ and $T^{+}$behavior of $G_{t}\left(T^{+}\right)$, we find

$$
G_{s}(u) \sim \frac{1}{s+u \frac{\left.\frac{\partial\left\langle t_{x_{0}}^{+}\right\rangle}{\partial x_{0}}\right|_{x_{0}=0}}{\left.\frac{\partial\left\langle t_{x_{0}}^{+}\right\rangle}{\partial x_{0}}\right|_{x_{0}=0}-\left.\frac{\partial\left\langle t_{x_{0}}\right\rangle}{\partial x_{0}}\right|_{x_{0}=0}}} .
$$

The differential equation for $\left\langle t_{x_{0}}^{+}\right\rangle$is well known and is obtained from the small $s$ expansion of Eq. (5)

$$
D\left[\frac{\partial^{2}}{\partial x_{0}^{2}}\left\langle t_{x_{0}}^{+}\right\rangle+\frac{F\left(x_{0}\right)}{k_{B} T} \frac{\partial}{\partial x_{0}}\left\langle t_{x_{0}}^{+}\right\rangle\right]=-1 .
$$

Solving this equation, using a similar equation for $\left\langle t_{x_{0}}^{-}\right\rangle$, and inverting Eq. (12) to the time domain we find the expected ergodic behavior

$$
G_{t}\left(T^{+}\right) \sim \delta\left(T^{+}-P_{B}^{+} t\right)
$$

where $P_{B}^{+}$is Boltzmann's probability of occupying $x>0$

$$
P_{B}^{+}=\frac{\int_{0}^{\infty} e^{-\frac{U(x)}{k_{B} T}} \mathrm{~d} x}{Z},
$$

$Z=\int_{-\infty}^{\infty} \exp -\frac{U(x)}{k_{B} T} \mathrm{~d} x$ is the normalizing partition function and $U(x)$ is the binding potential, with $F(x)=$ $-\mathrm{d} U(x) / \mathrm{d} x$.

Case 2 We consider the case where both the left and the right random walks are non recurrent. The survival probabilities in the two domains are $\epsilon_{x_{0}}^{+}$and $\epsilon_{x_{0}}^{-}$, in the long time limit. Then using Eqs. (79) we find for $t \rightarrow \infty$

$$
G_{t}\left(T^{+}\right) \sim \alpha^{-} \delta\left(T^{+}\right)+\alpha^{+} \delta\left(T^{+}-t\right)
$$

where

$$
\alpha^{+}=\frac{\left.\frac{\partial \epsilon_{x_{0}}^{+}}{\partial x_{0}}\right|_{x_{0}=0}}{\left.\frac{\partial \epsilon_{x_{0}}^{+}}{\partial x_{0}}\right|_{x_{0}=0}-\left.\frac{\partial \epsilon_{x_{0}}^{-}}{\partial x_{0}}\right|_{x_{0}=0}}
$$

and $\alpha^{-}=1-\alpha^{+}$. Since the particle always manages to escape either to the left or to the right, eventually the particle will either reside in the left domain or the right domain forever, hence the delta functions in Eq. (16). The weights of these delta functions are given by the derivatives of the survival probabilities only.

Case 3 We now consider a case where both the left and the right random walks are recurrent, though the average first return time from $x_{0}$ to $x=0$ is infinite, in such a way that Eq. (10) is valid. Then in the small $s$ and $u$ limit

$$
G_{s}(u) \sim \frac{s^{-1 / 2}+\mathcal{R}(s+u)^{-1 / 2}}{s^{1 / 2}+\mathcal{R}(s+u)^{1 / 2}}
$$

where the asymmetry parameter is

$$
\mathcal{R}=-\frac{\left.\frac{\partial A_{x_{0}}^{+}}{\partial x_{0}}\right|_{x_{0}=0}}{\left.\frac{\partial A_{x_{0}}^{-}}{\partial x_{0}}\right|_{x_{0}=0}} .
$$

Transforming to the time domain we find the asymmetric arcsine PDF [6]

$$
G_{t}\left(T^{+}\right) \sim \frac{1}{t} f\left(\frac{T^{+}}{t}\right)
$$

where

$$
f(x)=\frac{1}{\pi} \frac{\mathcal{R}}{x^{1 / 2}(1-x)^{1 / 2}\left[\mathcal{R}^{2}(1-x)+x\right]} .
$$

When $\mathcal{R}=1$ we find the arcsine law. Note that the PDF Eq. (20) diverges on $T^{+} / t=1$ and $T^{+} / t=0$, hence events where the particle always occupies (or hardly never occupies) the domain $x>0$ have a significant contribution.

Another general result obtained from the small $u$ expansion of Eq. (7) is for the average occupation time

$$
\left\langle T^{+}\right\rangle=\mathcal{L}_{s \rightarrow t}^{-1}\left\{\frac{J^{+}(s)}{s^{2}\left[J^{+}(s)-J^{-}(s)\right]}\right\}
$$

where $\mathcal{L}_{s \rightarrow t}^{-1}$ is the inverse Laplace transform. If the potential field is binding and the random walk is recurrent, then $\left\langle T^{+}\right\rangle \sim P_{B}^{+} t$. Similar relations between higher order moments of the occupation times and the survival probabilities are obtained in a similar way. In Sec. IV we consider several particular examples, which explain in greater detail the meaning of the general results obtained in this section. First we generalize our results for fractional dynamics.

\section{ANOMALOUS DIFFUSION}

Anomalous diffusion and relaxation is modeled based on the fractional time Fokker-Planck equation (FFPE) [17, 18], the concentration of non-interacting particles obeys

$$
\frac{\partial^{\alpha} c(x, t)}{\partial t^{\alpha}}=D_{\alpha}\left[\frac{\partial^{2}}{\partial x^{2}}-\frac{\partial}{\partial x} \frac{F(x)}{k_{b} T}\right] c(x, t),
$$

where $D_{\alpha}$ is a generalized diffusion coefficient and $0<$ $\alpha<1$. A brief mathematical introduction to the FFPE is given in Appendix A. We recall physical properties of the FFPE. (i) when $F(x)=0$ and for free boundary conditions we have the fractional diffusion equation 30, 31, 32, 33, 34] with anomalous diffusion $\left\langle x^{2}\right\rangle \propto$ $t^{\alpha}$. (ii) In the presence of a binding time independent force field the equilibrium is the Boltzmann distribution [17, [18]. (iii) Generalized Einstein relations are satisfied in consistency with linear response theory [17, 18]. (iv) Relaxation of modes follows the Mittag Leffler decay, related for example to Cole-Cole relaxation [17, 18]. (v) In the limit $\alpha \rightarrow 1$ we recover the standard Smoluchowski Fokker-Planck equation. The FFPE is derived from the continuous time random walk 18. Its mathematical foundation is P. Lévy's generalized central limit 
theorem applied to the number of steps in the underlying random walk [35, 36]. A very general solution of the FFPE in terms of the solution of the standard $\alpha=1$ Fokker-Planck equation was given in [35] (i.e., subordination, and the inverse Lévy transform). Recently there is some controversy on how to apply boundary conditions [37, 38, 39] for the anomalous case. Applications of fractional diffusion modeling include: Scher-Montroll time of flight transport of charge carriers in disordered medium [35], dynamics of ion channels [40], relaxation processes in proteins [41], and dielectric relaxation [4], and deterministic diffusion [32]. For a review and a popular article on fractional kinetics see [15, 16].

Similar to the normal diffusion case, we define $P_{x_{0}, t}\left(T^{+}\right)$as the PDF of $T^{+}$and $P_{x_{0}, s}(u)$, its double Laplace transform. As we show in next subsection the differential Eq. for $P_{x_{0}, s}(u)$ for the dynamics described by the FFPE Eq. (23) is

$$
\begin{gathered}
D_{\alpha}\left[\frac{\partial^{2}}{\partial x_{0}^{2}}+\frac{F\left(x_{0}\right)}{k_{b} T} \frac{\partial}{\partial x_{0}}\right] P_{x_{0}, s}(u)- \\
{\left[s+\Theta\left(x_{0}\right) u\right]^{\alpha} P_{x_{0}, s}(u)=-\left[s+\Theta\left(x_{0}\right) u\right]^{\alpha-1} .}
\end{gathered}
$$

The boundary conditions for Eq. (24) are identical to the normal diffusion case $\alpha=1$ given in Eq. (44). Eq. (24) is important since as pointed out in [19], fractional dynamics is weakly non-ergodic [43], namely occupation time statistics is not described by Boltzmann equilibrium even in the limit of long time and for binding potential fields. Thus the FFPE (23) cannot be used to describe time averages of physical observable due to ergodicity breaking, and the interpretation of results derived from the FFPE must be treated with care. Eq. (24) is a remedy for this problem since as we will show it yields a fractional framework for the calculation of non-trivial distribution of occupation times [i.e. generalization of Boltzmann's statistics Eq. (14)]. Eq. (24) is a fractional backward FokkerPlanck equation in double Laplace space, formally one may invert it to the time domain using material fractional derivatives [4], however in practice we solve this equation in double Laplace space and only then make the inverse double Laplace transform.

Interestingly the solution of the fractional Eq. (24) is identical to that found for normal diffusion case, namely our main results Eqs. (6178) are valid also in the nonMarkovian domain $0<\alpha<1$. Now $W_{x_{0}}^{ \pm}(s)$ needed for the calculation of $J^{ \pm}(s)$, is the Laplace transform of the survival probability for the fractional particle. Thus Eqs. (6 78) have some general validity beyond normal Markovian diffusion.

To prove that Eqs. (68) are still valid we must first find the differential equation for $W_{x_{0}}^{+}(s)$ : the survival probability of a fractional particle starting on $x_{0}>0$ in the domain $x>0$. We can prove that

$$
D_{\alpha}\left[\frac{\partial^{2}}{\partial x_{0}^{2}}+\frac{F\left(x_{0}\right)}{k_{B} T} \frac{\partial}{\partial x_{0}}\right] W_{x_{0}}^{+}(s)-s^{\alpha} W_{x_{0}}^{+}(s)=-s^{\alpha-1},
$$

and a similar equation holds for $x_{0}<0$. Eq. (25) is the fractional generalization of Tachiya's Eq. (5). The derivation of Eq. 25) is based on results obtained in 35] and is simple once the sub-ordination trick is used (see some details in Appendix A). Now using Eq. (25) it is easy to verify that Eqs. (68) are solutions of the fractional Eq. (24).

\section{A. Derivation of Fractional Equation for Occupation Times}

In this subsection we derive our main result Eq. (24) using the assumption that the underlying dynamics is described by the fractional Fokker-Planck equation (23). The latter describes long time behavior of the continuous time random walk (CTRW), which is the underlying random walk process we have in mind. In the CTRW a particle performs a one dimensional random walk on a lattice, with jumps to nearest neighbors only and with random waiting times between jumps. In CTRW the waiting times between jumps are independent identically distributed random variables, namely the CTRW process is renewed after each jump. The PDF of waiting times is $\psi(t)$. Two classes of CTRWs are usually considered, the case when the average waiting time is finite, and the case when $\psi(t) \propto t^{-(1+\alpha)}$ when $t \rightarrow \infty$ and $0<\alpha<1$. The latter case leads to a non-stationary behavior, aging, anomalous diffusion and weak ergodicity breaking [43]. The lattice spacing is $\epsilon$, eventually we will consider the continuum limit when $\epsilon$ is small. On each lattice point we assign a probability for jumping left and a probability of jumping right. This dynamics in the continuum limit leads to behavior described by the FFPE, when detailed balance conditions are applied on the probabilities for jumping left or right [18] (i.e. probabilities to jump left or right are related to external force field and temperature). In what follows we start with some general arguments, assuming only a renewal property of the random walk, without limiting our selves to a specific model.

The random position of the particle is $x(t)$. The total time the particle spends on $x \geq 0$ is $T^{+}$, i.e. the occupation time of half space. The particle starts on $x_{0}$ and assume that $x_{0} \geq 0$, later we generalize our results to the case $x_{0}<0$. We define the PDF of first passage times, from $x_{0}$ to $x=-\epsilon$, as $\psi_{x_{0}}^{+}(t)$. The PDF of first passage times from $x=0$ to $x=-\epsilon(x=-\epsilon$ to $x=0)$ is denoted with $\psi^{+}(t)$ and $\left[\psi^{-}(t)\right]$ respectively.

We assume that the first passage times PDFs $\psi^{+}(t)$ and $\psi^{-}(t)$ do not depend on $x_{0}$ and that sojourn times in domain $x>0$ and $x<0$ are statistically independent. Such assumption holds for Markovian dynamics but is not obvious otherwise. For CTRW dynamics the assumption is correct, since as mentioned the CTRW processes is a renewal process. The process is mapped on a two state process

$$
\theta_{x}(t)= \begin{cases}1 & x(t) \geq 0 \\ 0 & x(t)<0\end{cases}
$$


and hence $T^{+}=\int_{0}^{t} \theta_{x}(t) \mathrm{d} t$. Since either the particle is in the domain $x<0$ or not the dynamics is described by a set of sojourn times

$$
\tau_{x_{0}}, \tau_{1}^{-}, \tau_{2}^{+}, \tau_{3}^{-}, \tau_{4}^{+} \cdots
$$

Here the PDF of $\tau_{x_{0}}$ is $\psi_{x_{0}}(t)$, the PDF of $\tau_{1}^{-}$is $\psi^{-}(t)$, the PDF of $\tau_{2}^{+}$is $\psi^{+}(t)$, etc. All the sojourn times are assumed mutually independent, which means the process is renewed once the particle jumps from $x=0$ to $x=-\epsilon$ or vice versa.

Let $f_{x_{0}, t}\left(T^{+}\right)$be the PDF of $T^{+}$when the total observation time is $t$. Let $f_{x_{0}, s}(u)$ be the double Laplace transform of $f_{x_{0}, t}\left(T^{+}\right)$. A calculation, using methods of renewal theory, similar to the work of Godreche and Luck [9] yields

$$
f_{x_{0}, s}(u)=\frac{1-\psi_{x_{0}}^{+}(s+u)}{s+u}+\psi_{x_{0}}^{+}(s+u)\left[\psi^{-}(s) \frac{1-\psi^{+}(s+u)}{s+u}+\frac{1-\psi^{-}(s)}{s}\right] \frac{1}{1-\psi^{+}(s+u) \psi^{-}(s)} .
$$

Where $\psi_{x_{0}}^{+}(s+u)=\int_{0}^{t} \exp [-(s+u) t] \psi_{x_{0}}^{+}(t) \mathrm{d} t$ is the Laplace transform. If $\psi_{x_{0}}^{+}(t)=\psi^{+}(t)=\psi^{-}(t)$ we recover a result in [9]. If the particle starts on $x_{0}<0$ then one can show

$$
f_{x_{0}, s}(u)=\frac{1-\psi_{x_{0}}^{-}(s)}{s}+\psi_{x_{0}}^{-}(s)\left[\psi^{+}(s+u) \frac{1-\psi^{-}(s)}{s}+\frac{1-\psi^{+}(s+u)}{s+u}\right] \frac{1}{1-\psi^{+}(s+u) \psi^{-}(s)} .
$$

We now consider the case when the underlying dynamics is described by the FFPE. By definition the first passage time PDFs are related to survival probabilities according to

$$
W_{x_{0}}^{+}(t)=1-\int_{0}^{t} \psi_{x_{0}}^{+}(t) \mathrm{d} t
$$

or using the convolution theorem in Laplace space

$$
W_{x_{0}}^{+}(s)=\frac{1-\psi_{x_{0}}^{+}(s)}{s} .
$$

Hence we may rewrite Eq. (27)

$$
f_{x_{0}, s}(u)=W_{x_{0}}^{+}(s+u)+\left[1-(s+u) W_{x_{0}}^{+}(s+u)\right]\left[\psi^{-}(s) \frac{1-\psi^{+}(s+u)}{s+u}+\frac{1-\psi^{-}(s)}{s}\right] \frac{1}{1-\psi^{+}(s+u) \psi^{-}(s)} .
$$

Notice that $f_{x_{0}, s}(u)$ depends on $x_{0}$ only through the survival probability $W_{x_{0}}^{+}(s+u)$. If we apply the backward Fokker-Planck operator

$$
D_{\alpha}\left[\frac{\partial^{2}}{\partial x_{0}^{2}}+\frac{F\left(x_{0}\right)}{k_{B} T} \frac{\partial}{\partial x_{0}}\right]
$$

on this equation and use Eq. (25), namely we assume that the underlying dynamics is described by the FFPE in the continuum limit, we obtain at once our main result Eq. (24). Similar method is used for the case $x_{0}<0$ to complete the proof.

We now derive our main Eq. (7) using the continuum approximation. We consider the case when the particle start on $x_{0}=0$ hence we have $\psi_{x_{0}}^{+}=\psi^{+}$and define

$$
\bar{G}_{s}(u)=f_{x_{0}=0, s}(u) .
$$

Generally $\bar{G}_{s}(u)$ is not identical to $G_{s}(u)$ and our aim now is to find the conditions when these two functions are identical. Using (27) we have

$$
\begin{gathered}
\bar{G}_{s}(u)=\left[\frac{1-\psi^{+}(s+u)}{(s+u)}+\right. \\
\left.\psi^{+}(s+u) \frac{1-\psi^{-}(s)}{s}\right] \frac{1}{1-\psi^{+}(s+u) \psi^{-}(s)} .
\end{gathered}
$$

In the continuum limit we have the following $\epsilon$ expansion

$$
\psi_{\epsilon}^{-}(s) \simeq \psi_{\epsilon=0}^{-}(s)-\left.\frac{\partial \psi_{\epsilon}^{-}}{\partial x}\right|_{\epsilon=0} \epsilon+\cdots
$$

and a similar expansion holds for $\psi_{\epsilon}^{+}(s)$. Where the subscript $\epsilon$ in Eq. (34) is added to emphasize that the PDF of the first passage time from lattice point $-\epsilon$ to the origin 0 . Note that $\psi_{\epsilon=0}^{-}(s)=1$, since the particle on the origin is immediately absorbed. Inserting the expansion (34) in Eq. (33) and using a similar expansion for $\psi_{\epsilon}^{+}(s+u)$ we 
find that when $\epsilon \rightarrow 0$

$$
\bar{G}_{s}(u) \sim \frac{\left.\frac{1}{(s+u)} \frac{\partial \psi^{+}(s+u)}{\partial x_{0}}\right|_{x_{0}=0}-\left.\frac{1}{s} \frac{\partial \psi^{-}(s)}{\partial x_{0}}\right|_{x_{0}=0}}{\left.\frac{\partial \psi^{+}(s+u)}{\partial x_{0}}\right|_{x_{0}=0}-\left.\frac{\partial \psi^{-}(s)}{\partial x_{0}}\right|_{x_{0}=0}}
$$

Using Eqs. (30 35) we obtain our main result Eq. (17) and $\bar{G}_{s}(u)$ is identical to $G_{s}(u)$ in the continuum limit of $\epsilon \rightarrow 0$.

\section{EXAMPLES}

\section{A. Weak Ergodicity Breaking}

We now consider anomalous dynamics in a binding potential field $U(x)$, e.g. $U(x)=k x^{2} / 2$ with $k>0$, and $F(x)=-\mathrm{d} U(x) / \mathrm{d} x$. As we showed already the long time behavior of $G_{t}\left(T^{+}\right)$, for normal diffusion, yields an ergodic behavior Eq. (14). Residence time statistics for sub-diffusion in a binding potential field was considered previously in [19], using the continuous time random walk model on a lattice. Here we consider a fractional FokkerPlanck equation approach showing that concepts of weak ergodicity breaking in [19] are valid also within the fractional framework.

In 35] it was shown that the random walks in a binding field are recurrent for $\alpha<1$, just like the normal case $\alpha=1$. Using the subordination trick (see Appendix), or analyzing Eq. (25) we find that for $s \rightarrow 0$

$$
W_{x_{0}}^{ \pm}(s) \sim \frac{\left(\tau_{x_{0}}^{ \pm}\right)^{\alpha}}{s^{1-\alpha}} .
$$

When $\alpha=1$ we have $\left(\tau_{x_{0}}^{ \pm}\right)^{\alpha}=\left\langle t_{x_{0}}^{ \pm}\right\rangle$and the behavior in Eq. (11). For $0<\alpha<1$ we have in the time domain $W_{x_{0}}^{ \pm}(t) \propto t^{-\alpha}$, reflecting the long tailed trapping times of the underlying CTRW. The $\left(\tau_{x_{0}}^{ \pm}\right)^{\alpha}$ are amplitudes which satisfy

$$
D_{\alpha}\left[\frac{\partial^{2}}{\partial x_{0}{ }^{2}}+\frac{F\left(x_{0}\right)}{k_{B} T} \frac{\partial}{\partial x_{0}}\right]\left(\tau_{x_{0}}^{ \pm}\right)^{\alpha}=-1 .
$$

This equation is obtained from the small $s$ expansion of Eq. (25). Using Eq. (36) and Eq. (77) we find in the limit of small $s$ and $u$

$$
G_{s}(u) \sim \frac{\mathcal{R}(s+u)^{\alpha-1}+s^{\alpha-1}}{\mathcal{R}(s+u)^{\alpha}+s^{\alpha}}
$$

where the asymmetry parameter is

$$
\mathcal{R}=-\frac{\left.\frac{\partial\left(\tau^{+}\right)^{\alpha}}{\partial x_{0}}\right|_{x_{0}=0^{+}}}{\left.\frac{\partial\left(\tau^{-}\right)^{\alpha}}{\partial x_{0}}\right|_{x_{0}=0^{-}}} .
$$

Inverting to the time domain, we see that the PDF of $T^{+}$ in the long time $t$ limit is described by Lamperti's limit theorem $\underline{6}$ ]

$$
G_{t}\left(T^{+}\right) \sim \frac{1}{t} \delta_{\alpha}\left(\mathcal{R}, \frac{T^{+}}{t}\right) .
$$

where the scaling function is

$$
\begin{gathered}
\delta_{\alpha}(\mathcal{R}, p) \equiv \\
\frac{\sin \pi \alpha}{\pi} \frac{\mathcal{R} p^{\alpha-1}(1-p)^{\alpha-1}}{\mathcal{R}^{2}(1-p)^{2 \alpha}+p^{2 \alpha}+2 \mathcal{R}(1-p)^{\alpha} p^{\alpha} \cos \pi \alpha} .
\end{gathered}
$$

This function is normalized according to $\int_{0}^{1} \delta_{\alpha}(\mathcal{R}, p) \mathrm{d} p=1$. When $\alpha=1$ we find the ergodic behavior in Eq. (14), while clearly if $\alpha<1$ we find a non-ergodic behavior. The parameter $\mathcal{R}$ is called the asymmetry parameter. It can be calculated solving Eq. (37), we find

$$
\begin{aligned}
& \left.\frac{\partial\left(\tau_{x_{0}}^{+}\right)^{\alpha}}{\partial x_{0}}\right|_{x_{0}=0^{+}}=\frac{1}{D_{\alpha}} \int_{0}^{\infty} e^{-\left[U\left(x^{\prime}\right)-U(0)\right] / k_{b} T} \mathrm{~d} x^{\prime}, \\
& \left.\frac{\partial\left(\tau_{x_{0}}^{-}\right)^{\alpha}}{\partial x_{0}}\right|_{x_{0}=0^{-}}=-\frac{1}{D_{\alpha}} \int_{-\infty}^{0} e^{-\left[U\left(x^{\prime}\right)-U(0)\right] / k_{b} T} \mathrm{~d} x^{\prime} .
\end{aligned}
$$

Using Eqs. 394243 we find

$$
\mathcal{R}=\frac{P_{B}^{+}}{1-P_{B}^{+}},
$$

where $P_{B}^{+}$is Boltzmann's probability of finding the particle in the domain $x>0$ Eq. (15). Eqs. (4044) were found previously in 19] using a different approach. One can show that the average occupation time is

$$
\left\langle T^{+}\right\rangle \sim P_{B}^{+} t
$$

and fluctuation are very large if $\alpha<1$

$$
\left\langle T^{+2}\right\rangle-\left\langle T^{+}\right\rangle^{2} \sim(1-\alpha) P_{B}^{+}\left(1-P_{B}^{+}\right) t^{2} .
$$

We inrtoduce a measure for ergodicity breaking the EB parameter

$$
\mathrm{E} B=\frac{\left\langle T^{+2}\right\rangle-\left\langle T^{+}\right\rangle^{2}}{\left\langle T^{+}\right\rangle^{2}} \sim(1-\alpha) \frac{1-P_{B}^{+}}{P_{B}^{+}},
$$

which is zero in the ergodic phase $\alpha=1$.

\section{B. Diffusion in an interval}

We consider the case where the particle is free to diffuse in an interval of total length $L^{+}+L^{-}$. The particle is initially on the origin $x=0$ and reflecting boundary conditions are on $x=L^{+}$and $x=-L^{-}$. Statistical properties of $T^{+}$the time spent in $\left(0, L^{+}\right)$are now investigated. 25

The survival probability can be calculated using Eq.

$$
W_{x_{0}}^{+}(s)=\frac{1-\frac{\cosh \left[\sqrt{\frac{s^{\alpha}}{D_{\alpha}}}\left(L^{+}-x_{0}\right)\right]}{\cosh \left(\sqrt{\frac{s^{\alpha}}{D_{\alpha}}} L^{+}\right)}}{s},
$$


and when $\alpha=1$ we recover a text book result [2]. Using Eq. (17) we find

$$
\begin{gathered}
G_{s}(u)= \\
\frac{(s+u)^{\alpha / 2-1} \tanh \left[\frac{(s+u)^{\alpha / 2} L^{+}}{\sqrt{D_{\alpha}}}\right]+s^{\alpha / 2-1} \tanh \left(\frac{s^{\alpha / 2} L^{-}}{\sqrt{D_{\alpha}}}\right)}{(s+u)^{\alpha / 2} \tanh \left[\frac{(s+u)^{\alpha / 2} L^{+}}{\sqrt{D_{\alpha}}}\right]+s^{\alpha / 2} \tanh \left(\frac{s^{\alpha / 2} L^{-}}{\sqrt{D_{\alpha}}}\right)} .
\end{gathered}
$$

For free boundary conditions, namely in the limit where the system size is infinite $L^{+} \rightarrow \infty$ and $L^{-} \rightarrow \infty$ we find

$$
G_{s}(u)=\frac{(s+u)^{\alpha / 2-1}+s^{\alpha / 2-1}}{(s+u)^{\alpha / 2}+s^{\alpha / 2}} .
$$

Inverting to the time domain, the $\mathrm{PDF}$ of $T^{+}$is the symmetric Lamperti PDF with index $\alpha / 2$

$$
G_{t}\left(T^{+}\right)=\frac{1}{t} \delta_{\alpha / 2}\left(1, \frac{T^{+}}{t}\right) .
$$

When $\alpha=1$, i.e. the case of normal Gaussian diffusion, we recover the well known arcsine distribution. As $\alpha$ is decreased we are more likely to find the particle localized in $x>0$ or $x<0$ for a time of the order of the observation time. Indeed when $\alpha \rightarrow 0$ the $\mathrm{PDF}$ of $T^{+}$is a combination of two delta functions with $T^{+}=t$ (particle always on $x>0$ ) or $T^{+}=0$ (particle always in $x<0$ ).

A different behavior is found for finite $L^{+}$and $L^{-}$, then using Eq. (49) we find

$$
G_{t}\left(T^{+}\right) \sim \frac{1}{t} \delta_{\alpha / 2}\left(1, \frac{T^{+}}{t}\right) \quad t<<\left[\frac{\min \left(L^{+}, L^{-}\right)^{2}}{D_{\alpha}}\right]^{1 / \alpha} .
$$

For these time scales the particle does not interact with the boundaries, and $G_{t}\left(T^{+}\right)$is the symmetric Lamperti $\mathrm{PDF}$ with index $\alpha / 2$. In the long time limit, corresponding to small $s u$ limit we find using Eq. (49)

$$
G_{s}(u) \sim \frac{(s+u)^{\alpha-1} L^{+}+s^{\alpha-1} L^{-}}{(s+u)^{\alpha} L^{+}+s^{\alpha} L^{-}}
$$

and hence when $t \rightarrow \infty$

$$
G_{t}\left(T^{+}\right) \sim \frac{1}{t} \delta_{\alpha}\left(\frac{L^{+}}{L^{-}}, \frac{T^{+}}{t}\right) .
$$

This is in agreement with our more general result Eqs. (4044) namely for the case of free diffusion $P_{B}^{+}=$ $L^{+} /\left(L^{+}+L^{-}\right)$and hence $\mathcal{R}=L^{+} / L^{-}$. If $L^{+} \neq L^{-}$ the PDF of $T^{+}$is as expected non-symmetric, reflecting the tendency of the particle to reside in the larger interval [say $\left(0, L^{+}\right)$if $L^{+}>L^{-}$] for longer times compared with the shorter domain. For long times an equilibrium is obtained: for $\alpha=1$ an ergodic phase is found where $T^{+} / t=L^{+} /\left(L^{-}+L^{+}\right)$while for $\alpha<1$ weak ergodicity breaking is found. We see that the statistics of occupation times exhibits a transition from a symmetric Lamperti PDF with index $\alpha / 2$ when diffusion is dominating the dynamics, i.e. for short times, to a generally nonsymmetric Lamperti PDF with index $\alpha$, for long times when the particle interacts with the boundaries. Such a transition is not limited to free diffusion as we show later.

If $L^{-} \rightarrow \infty$ while $L^{+}$remains finite a different behavior is found. Now the particle can be found either in a domain of finite length $0<x<L^{+}$or in the infinite domain $-\infty<x<0$. Statistically we expect of-course that the particle will reside more in $x<0$, though the random walk is recurrent hence, after each sojourn time in $x<0$ the particle is ejected back to $x>0$, provided that we wait long enough. However the average return time from a point in $x<0$ to some point in $x>0$ is infinite, and this means that simple scaling $T^{+} \sim t$ does not not hold. For this case we have

$$
G_{s}(u)=\frac{(s+u)^{\alpha / 2-1} \tanh \left[\frac{(s+u)^{\alpha / 2} L^{+}}{\sqrt{D_{\alpha}}}\right]+s^{\alpha / 2-1}}{(s+u)^{\alpha / 2} \tanh \left[\frac{(s+u)^{\alpha / 2} L^{+}}{\sqrt{D_{\alpha}}}\right]+s^{\alpha / 2}} .
$$

To investigate deviations from simple scaling we consider moments of the random variable $T^{+}$, using the small $u$ expansion of Eq. (55). The average occupation time in $0<x<L^{+}$is

$$
\left\langle T^{+}\right\rangle=\mathcal{L}_{s \rightarrow t}^{-1}\left[\frac{1}{2 s^{2}}\left(1-e^{-\frac{2 s^{\alpha} / 2_{L}}{\sqrt{D_{\alpha}}}}\right)\right],
$$

where $\mathcal{L}_{s \rightarrow t}^{-1}$ is the inverse Laplace transform. This expression is inverted using one sided Lévy stable functions, recall

$$
l_{\alpha / 2,2 L^{+} / \sqrt{D_{\alpha}}, 1}(t)=\mathcal{L}_{s \rightarrow t}^{-1} e^{-2 s^{\alpha / 2} L^{+} / \sqrt{D_{\alpha}}},
$$

and see 35] and Ref. therein for more mathematical details on this function. The one sided stable cumulative distribution is

$$
L_{\alpha / 2, \frac{2 L^{+}}{\sqrt{D_{\alpha}}}, 1}(t)=\int_{0}^{t} l_{\alpha / 2, \frac{2 L^{+}}{\sqrt{D_{\alpha}}}, 1}(t) \mathrm{d} t,
$$

and hence

$$
\left\langle T^{+}\right\rangle=\frac{1}{2} \int_{0}^{t}\left[1-L_{\alpha / 2, \frac{2 L^{+}}{\sqrt{D_{\alpha}}}, 1}(t)\right] \mathrm{d} t .
$$

For short times $\left\langle T^{+}\right\rangle=t / 2$, since then the particle does not have time to interact with the boundary, and it spends half of the time in $x>0$. For long times

$$
\left\langle T^{+}\right\rangle \sim \frac{L^{+}}{\sqrt{D_{\alpha}}} \frac{t^{1-\alpha / 2}}{\Gamma(2-\alpha / 2)} .
$$

We see that as the process becomes slower, namely when $\alpha$ is decreased, the particle tends to stay more in $0<$ $x<L^{+}$i.e. $\left\langle T^{+}\right\rangle \propto t$ for $\alpha=0$ but $\left\langle T^{+}\right\rangle \propto t^{1 / 2}$ if $\alpha=1$. We explain this result for normal diffusion $\alpha=1$ 
by thinking about the process as a two state process, i.e. the particle is either in $x<0$ or in $x>0$. Sojourn times in $x>0$ are finite, since the interval $0<x<L^{+}$is finite. The PDF of times in state $x<0$ follow the $t^{-3 / 2}$ power law tail due to usual diffusion. The number of time the particle will cross zero is $\langle n(t)\rangle \sim t^{1 / 2}$ at-least for a lattice CTRW process (in the continuum limit this question is not well defined). Hence we expect $\left\langle T^{+}\right\rangle=$ $\langle n(t)\rangle *$ average time in $0<x<L^{+} \sim t^{1 / 2}$ as we find. If $\alpha<1$ we expect $\langle n(t)\rangle \sim t^{\alpha / 2}$ and the average time is $0<$ $x<L^{+}$is proportional to $\int^{t} \psi(t) t \mathrm{~d} t \simeq \int^{t} t^{-(1+\alpha)} t \mathrm{~d} t \simeq$ $t^{1-\alpha}$, hence we get $\left\langle T^{+}\right\rangle \propto t^{1-\alpha / 2}$. The second moment of $T^{+}$is

$$
\left\langle T^{+2}\right\rangle \sim 4(1-\alpha) \frac{L^{+}}{\sqrt{D_{\alpha}}} \frac{t^{2-\alpha / 2}}{\Gamma(3-\alpha / 2)} .
$$

Note that we do not have simple scaling and $\left\langle T^{+2}\right\rangle \propto$ $t^{2-\alpha / 2}$ is not proportional to $\left\langle T^{+}\right\rangle^{2} \propto t^{2-\alpha}$, and hence the PDF of $T^{+}$does not have a simple scaling.

\section{Diffusion with Drift}

We consider anomalous diffusion in the presence of a constant driving force $F>0$, for an infinite system. The biased diffusion yields a net drift $\langle x\rangle=D_{\alpha} F t^{\alpha} /\left[k_{b} T \Gamma(1+\right.$ $\alpha)$ ]. Since $F>0$ the particle will escape to infinity, hence for a particle starting on $x=0$ we expect $T^{+} \sim t$ when $t$ is large.

The survival probability in the right half space

$$
W_{x_{0}}^{+}(s)=\frac{1-\exp \left[-\frac{F \beta^{+}(s) x_{0}}{2 k_{b} T}\right]}{s},
$$

when $x_{0}>0$. To obtain the survival probability for left random walks replace $\beta^{+}(s)$ in Eq. (62) with $\beta^{-}(s)$, and

$$
\beta^{ \pm}(s)=1 \pm \sqrt{1+4 s^{\alpha} \tau^{\alpha}}
$$

where

$$
\tau^{\alpha}=\frac{\left(k_{b} T\right)^{2}}{F^{2} D_{\alpha}}
$$

Using Eq. (8)

$$
G_{s}(u)=\frac{\frac{\beta^{+}(s+u)}{(s+u)}-\frac{\beta^{-}(s)}{s}}{\beta^{+}(s+u)-\beta^{-}(s)} .
$$

For $s^{\alpha} \tau^{\alpha}>>1$ and $u^{\alpha} \tau^{\alpha}>>1$

$$
G_{s}(u) \simeq \frac{(s+u)^{\alpha / 2-1}+s^{\alpha / 2-1}}{(s+u)^{\alpha / 2}+s^{\alpha / 2}} .
$$

Thus for short times $t<<\tau$

$$
G_{t}\left(T^{+}\right) \simeq \frac{1}{t} \delta_{\alpha / 2}\left(1, \frac{T^{+}}{t}\right)
$$

namely a symmetric Lamperti PDF with index $\alpha / 2$ describes the residence times. Such behavior is independent of the drift and can be understood if we notice that for short times the dynamics is governed by diffusion not drift. To see this recall that the scaling of these two processes is $x \sim t^{\alpha / 2}$ (diffusion) and $x \sim t^{\alpha}$ (drift) and hence for short times diffusion wins. For long times we use the small $s, u$ expansion of Eq. (65), $G_{s}(u) \sim 1 /(s+u)$ which gives the expected behavior $G_{t}\left(T^{+}\right) \sim \delta\left(T^{+}-t\right)$.

The mean occupation time is

$$
\left\langle T^{+}\right\rangle=\mathcal{L}_{s \rightarrow t}^{-1}\left\{\frac{1}{2 s^{2}} \frac{1+\sqrt{1+4 s^{\alpha} \tau^{\alpha}}}{\sqrt{1+4 s^{\alpha} \tau^{\alpha}}}\right\} .
$$

For the case of normal diffusion $\alpha=1$ we find

$$
\left\langle T^{+}\right\rangle=\frac{t}{2}+\tau\left[\sqrt{\frac{t}{\pi \tau}} e^{-\frac{t}{4 \tau}}+\left(\frac{t}{2 \tau}-1\right) \operatorname{Erf} \sqrt{\frac{t}{4 \tau}}\right] .
$$

The long time behavior is

$$
\left\langle T^{+}\right\rangle \sim t-\tau\left(1-\sqrt{\frac{4 \tau}{\pi t}} e^{-\frac{t}{4 \tau}}\right),
$$

the leading term $\left\langle T^{+}\right\rangle \sim t$ is expected since as mentioned for long times the particle is always in $x>0$ when $F>0$. For short times

$$
\left\langle T^{+}\right\rangle \sim \frac{t}{2}\left[1+\frac{2}{3 \sqrt{\pi}}\left(\frac{t}{\tau}\right)^{1 / 2}-\frac{1}{30 \sqrt{\pi}}\left(\frac{t}{\tau}\right)^{3 / 2}+0\left(t^{5 / 2}\right)\right] .
$$

The leading term $\left\langle T^{+}\right\rangle \sim \frac{t}{2}$ shows that at short time diffusion not drift is dominating the process, hence from symmetry half of the time the particle is on $x>0$. For the sub-diffusive case $\alpha<1$ we investigate the long time behavior of $\left\langle T^{+}\right\rangle$using the small $s$ expansion of (68) and then inverting to the time domain

$$
\left\langle T^{+}\right\rangle \sim t\left[1-\frac{1}{\Gamma(2-\alpha)} \frac{\left(k_{b} T\right)^{2}}{F^{2} D_{\alpha} t^{\alpha}}+\mathrm{O}\left(\frac{\tau}{t}\right)^{2 \alpha}\right] .
$$

At short times we use Eq. (68) and Hankel's contour integral, for the $\Gamma(z)$ function, and find

$$
\left\langle T^{+}\right\rangle \sim \frac{t}{2}\left[1+\frac{1}{2 \Gamma(2+\alpha / 2)} \frac{F \sqrt{D_{\alpha}}}{\left(k_{b} T\right)^{2}} t^{\alpha / 2}+\cdots\right] .
$$

We note that results for the case $F<0$ can be easily obtained from our results for $F>0$. The distribution of times $T^{-}$in $x<0$ when $F>0$ is equal of-course to the distribution of time $T^{+}$in $x>0$ when $F<0$. Also $T^{+}+T^{-}=t$ hence a simple shift of the random variable yields $T^{-}=t-T^{+}$, and hence also statistics for the case $F<0$.

\section{Diffusion in an Unstable Force Field}

We consider a particle in an unstable force field

$$
F(x)=\left\{\begin{array}{cc}
F_{+} & x>0 \\
-F_{-} & x<0
\end{array}\right.
$$


where $F_{+}>0$ and $F_{-}>0$. For this case the particle will eventually escape either to $+\infty$ or $-\infty$, and the random walk is not recurrent. The survival probabilities in left and right domains are

$$
W_{x_{0}}^{ \pm}(s)=\frac{1}{s}\left\{1-\exp \left[\mp F_{ \pm}\left(1+\sqrt{1+4 s^{\alpha} \tau_{ \pm}^{\alpha}}\right) x_{0} /\left(2 k_{b} T\right)\right]\right\} .
$$

where

$$
\tau_{ \pm}^{\alpha}=\frac{\left(k_{b} T\right)^{2}}{F_{ \pm}^{2} D_{\alpha}}
$$

Using Eq. (7) we find

$$
G_{s}(u)=\frac{\frac{\gamma_{+}(s+u)}{s+u}+\frac{\gamma_{-}(s)}{s}}{\gamma_{+}(s+u)+\gamma_{-}(s)}
$$

where

$$
\gamma_{ \pm}(s)=F_{ \pm}\left(1+\sqrt{1+4 s^{\alpha} \tau_{ \pm}^{\alpha}}\right) .
$$

Using the definition Eq. (9), the small $s$ behavior of Eq. (75) gives the survival probabilities in the + and domains, $x>0$ and $x<0$ respectively. In the limit of long times

$$
\epsilon_{ \pm}\left(x_{0}\right)=1-\exp \left(\mp \frac{F_{ \pm} x_{0}}{k_{b} T}\right),
$$

where $x_{0}>0$ for + and $x_{0}<0$ for - . Hence according to the rather general Eqs. (16 17) we find

$$
G_{t}\left(T^{+}\right) \sim \frac{F_{+}}{F_{-}+F_{+}} \delta\left(T^{+}-t\right)+\frac{F_{-}}{F_{+}+F_{-}} \delta\left(T^{+}\right) .
$$

This long time behavior exhibits the same behavior for the normal diffusion $\alpha=1$ as for the anomalous case $\alpha<1$. Note that Eqs. (16 17) where derived for normal diffusion however one can show that they are valid also for the anomalous diffusion case. To see this use the small $s, u$ expansion of Eq. (77) which gives

$$
G_{s}(u) \sim \frac{1}{F_{+}+F_{-}}\left(\frac{F_{+}}{s+u}+\frac{F_{-}}{s}\right),
$$

which is the double inverse Laplace transform of Eq. (80) and is independent of the parameters $\alpha, D_{\alpha}$ and $T$.

For short times $t \ll \min \left(\tau_{-}, \tau_{+}\right)$we use the large $s, u$ behavior of Eq. (77) and find $G_{t}\left(T^{+}\right) \simeq \frac{1}{t} \delta_{\alpha / 2}\left(1, \frac{T^{+}}{t}\right)$. Thus for short times the PDF of $T^{+}$is the symmetric Lamperti PDF which is independent of all the parameters of the problem except for $\alpha$. At the early stages of the dynamics the diffusion process not the drift is the most important, and hence forces are not relevant.

\section{E. Diffusion in Binding Force Field}

We consider a particle in a stable force field

$$
F(x)=\left\{\begin{array}{cc}
-F_{+} & x>0 \\
F_{-} & x<0
\end{array}\right.
$$

where $F_{+}>0$ and $F_{-}>0$. The random walk is recurrent. Using an approach similar to one used in previous subsection

$$
G_{s}(u)=\frac{\frac{\xi_{+}(s+u)}{s+u}+\frac{\xi_{-}(s)}{s}}{\xi_{+}(s+u)+\xi_{-}(s)},
$$

and $\xi_{ \pm}(s)=F_{ \pm}\left(1-\sqrt{1+4 s^{\alpha} \tau_{ \pm}^{\alpha}}\right)$.

For small $s, u$ we have

$$
G_{s}(u) \sim \frac{(s+u)^{\alpha-1} \frac{F_{-}}{F_{+}}+s^{\alpha-1}}{(s+u)^{\alpha} \frac{F_{-}}{F_{+}}+s^{\alpha}},
$$

and hence when $t$ is large

$$
G_{t}\left(t^{+}\right) \sim \frac{1}{t} \delta_{\alpha}\left(\frac{F_{-}}{F_{+}}, \frac{T^{+}}{t}\right) .
$$

This is in agreement with our more general results Eqs. (40 44). For short times we have $G_{t}\left(T^{+}\right) \simeq$ $\frac{1}{t} \delta_{\alpha / 2}\left(1, \frac{T^{+}}{t}\right)$ which is similar to the behavior of the unstable field discussed in previous sub-section.

\section{DISCUSSION}

Statistics of occupation times for binding external fields exhibits in the limit of long times an ergodic behavior when the diffusion is normal, or weak ergodicity breaking Eqs. (4044) when diffusion is anomalous. We established a link between weak ergodicity breaking and fractional calculus. The exponent $\alpha$ in the fractional derivative $\partial^{\alpha} / \partial t^{\alpha}$ enters in Eq. (40) describing the non-ergodic properties of the residence times. Since many processes and systems are modeled today using the fractional calculus approach, it is not out of the question that weak ergodicity breaking has many applications, and is wide spread. We can say that at-least one must treat with care, results obtained using fractional kinetic equations, since they describe only ensemble averages, not time averages.

For binding external fields our results are in full agreement with those derived recently, by Bel and the author [19]. There a continuous time random walk process was considered. Technically the methods used to treat the two problems are different. For the fractional framework, a differential equation, Eq. (24) for the occupation times is derived and solved, which yields the weakly non ergodic properties of the system, while for the CTRW certain recursive relations must be solved 20]. Our work shows how the fractional framework, which is the continuum limit of the CTRW (and in this sense simpler) can be used to obtain statistics of residence times, and for binding fields weak ergodicity breaking.

We found a general relation between the problem of occupation times and the problem of first passage times, Eq. (7). Mathematically the problem of first passage time is described in terms of a differential equation whose 
solution depends on a single time $t$ as a parameter, while the equation for residence time depends on two times $T^{+}$and $t$, hence the approach based on calculation of first passage times instead of a direct calculation of distribution of residence times seems to us useful. Besides, statistics of first passage times in external fields is a well investigated problem and this knowledge was used here. We showed that from Eq. (7) general properties of residence times can be easily derived. Luckily the relation Eq. (7) is valid for both the non-Markovian sub-diffusive and normal processes, and hence has some general validity. From our derivation of this relation, we see that a key ingredient for this relation to be valid is the renewal property of the underlying random walk. As far as we know statistics of residence times for more general non-Markovian and non renewal dynamics was not investigated so far [45].

As mentioned, for binding fields we confirm old results in the limit of long measurement times. New results for non-binding force fields and also for non asymptotic times are also found. For example, for the simplest case of anomalous diffusion in free space, we found that the PDF of occupation times in half space is a symmetric Lamperti PDF with index $\alpha / 2$. This is the natural generalization of the well known arcsine law. Hence we showed that the fractional kinetic framework is indeed a natural generalization of the ordinary diffusion process.

Other behaviors are found when diffusion is not free. For example for short times we expect rather generally that occupation times statistics is described by a symmetric Lamperti PDF, since for short times drift by force fields is a slow process if compared with the diffusion process. For long times we get either (i) weak ergodicity breaking if the force field is binding, or (ii) occupation times described by Eq. (16) if both the left and right random walks are not recurrent (note that this equation is also valid for anomalous sub diffusion), or (iii) when random walk in the left and right domain is not bounded and recurrent, in such a way that the average first return time is infinite, then a Lamperti PDF describes statistics of occupation times in the limit of long times. For normal diffusion process the asymmetry parameter is determined by Eq. (19). We also analyzed other cases where the left random walk is recurrent but not bounded and the right random walk is bounded, showing deviations from simple scaling.

Acknowledgment The Israel Science Foundation supported this work. I thank A. Comtet, S. Majumdar and G. Margolin, for comments on the manuscript, and Golan Bel for fruitful discussions and a previous collaboration, which led to the current research.

\section{APPENDIX A}

The Fokker-Planck equation for a particle in a force field reads

$$
\frac{\partial c(x, t)}{\partial t}=D_{1} L_{\mathrm{FP}} c(x, t)
$$

with the operator

$$
L_{\mathrm{FP}}=\frac{\partial^{2}}{\partial x^{2}}-\frac{\partial}{\partial x} \frac{F(x)}{k_{B} T},
$$

where $D_{1}$ is the diffusion coefficient. To prepare for the FFPE we rewrite Eq. [86) in an integral form

$$
c(x, t)-\delta\left(x-x_{0}\right)=D_{10} I_{t}^{1} L_{\mathrm{FP}} c(x, t)
$$

where $\delta\left(x-x_{0}\right)$ are the initial conditions. According to the fractional kinetic approach, we must replace the integral ${ }_{0} I_{t}^{1}$ with a fractional Riemann-Liouville integration, defined as an operation on a function $Z(t)$ according to

$$
{ }_{0} I_{t}^{\alpha} Z(t) \equiv \frac{1}{\Gamma(\alpha)} \int_{0}^{t} \frac{Z\left(t^{\prime}\right)}{\left(t-t^{\prime}\right)^{1-\alpha}} \mathrm{d} t^{\prime},
$$

and for our purpose $0<\alpha<1$. The FFPE in its integral form is

$$
c(x, t)-\delta\left(x-x_{0}\right)=D_{\alpha 0} I_{t}^{\alpha} L_{\mathrm{FP}} c(x, t)
$$

where $D_{\alpha}$ is a generalized diffusion coefficient. Several authors present this equation in different ways. Sometimes 17] further differentiation with respect to time is made in Eq. (90) to return to a fractional differential equation instead of the fractional integral form. However, following work of Gorenflo and Mainardi e.g. [34] there is now growing use of Caputo symbols which are more elegant. Such symbols are used in Eq. (23) which has the same meaning as Eq. (90). Later we use the Laplace $t \rightarrow s$ transform of the FFPE equation (90)

$$
s c(x, s)-\delta\left(x-x_{0}\right)=D_{\alpha} s^{1-\alpha} L_{\mathrm{FP}} c(x, s) .
$$

We see that the solution of FFPE with $\alpha<1$ is related to the solution of the Fokker-Planck equation when $\alpha=1$ in Laplace space. To obtain the solution of the FFPE from the solution of the usual Fokker-Planck equation we must make a replacement $D_{1} \rightarrow D_{\alpha} s^{1-\alpha}$ [17]. This similarity transformation in $s$ space can be inverted to real time [35], and with it one can obtain a solution of the FFPE once the corresponding solution of the usual Fokker-Planck equation is known. This transformation is related to subordination and the inverse Lévy transform [35].

Similarly the survival probability for the normal diffusion case, and the fractional case are related to each other, by a simple transformation in Laplace space [35]. According to Eq. (57) in Ref. 35]

$$
W_{\alpha, x_{0}}^{+}(s)=\frac{D_{1}}{D_{\alpha}} s^{\alpha-1} W_{1, x_{0}}^{+}\left(\frac{D_{1}}{D_{\alpha}} s^{\alpha}\right),
$$


where $W_{\alpha, x_{0}}^{+}(s)$ is the survival probability for the fractional $\alpha<1$ or the normal case $\alpha=1$. Using Eqs. (5.
92) it is easy to prove the validity of Eq. (25).
[1] S. N. Majumdar cond-mat/0510064

[2] S. Redner A Guide to First-Passage Processes Cambridge University Press 2001 Cambridge.

[3] D. A. Darling, and M. Kac, Trans. Am. Math. Soc. 84 444 (1957).

[4] G. H. Weiss Aspects and Applications of the Random Walk North Holland 1994 Amsterdam.

[5] A. M. Berezhkovskii, V. Zaloj, and N. Agmon Phys. Rev. E 573937 (1998).

[6] J. Lamperti, Trans. Amer. Math. Soc. 88380 (1958).

[7] O. Benichou, M. Coopey, M. Moreau, P. H. Suet, and R. Voituriez Europhysics Letters 7042 (2005)

[8] A. Truman, and D. Williams, Diffusion Processes and Related Problems in Analysis vol. 1 Pinsky editor, Birkhauser-Boston Inc. 1990.

[9] C. Godreche, and J. M. Luck, J. of Statistical Physics 104489 (2001).

[10] G. Margolin, E. Barkai Phys. Rev. Let. 94080601 (2005).

[11] G. Margolin, V. Protasenko, M. Kuno, E. Barkai Advances in Chemical Physics in production, cond-mat/0506512

[12] G. Bel, E. Barkai, nlin.CD/0507036 (submitted).

[13] R. Marathe, and A. Dhar Phys. Rev. E 72066112 (2005).

[14] S. N. Majumdar, and A. Comtet Phys. Rev. Lett. 89 060601 (2002).

[15] R. Metzler, J. Klafter, Phys. Rep. 3391 (2000).

[16] I. M. Sokolov, J. Klafter, A. Blumen Physics Today 55 48 (2002).

[17] R. Metzler, E. Barkai, and J. Klafter, Phys. Rev. Lett. 823563 (1999).

[18] E. Barkai, R. Metzler, J. Klafter, Phys. Rev. E 61132 (2000).

[19] G. Bel, E. Barkai, Phys. Rev. Lett. 94240602 (2005).

[20] G. Bel, E. Barkai, Phys. Rev. E in press and condmat/050633.

[21] A. Blumen, and G. Zumofen J. of Chem. Phys. 75892 (1981); 76, 3713 (1982).

[22] N. Agmon, J. Chem. Phys. 813644 (1984)

[23] A. Bar-Haim, and J. Klafter J. Chem. Phys. 1095187 (1998).
[24] E. Barkai, Y. Jung and R. Silbey Annual Review of Physical Chemistry 55, 457 (2004).

[25] C. Bustamante, J. Liphardt, and F. Ritort, Phys. Today 58(7) 43 (2005).

[26] G. Zumofen, J. Hohlbein, C. G Hubner, Phys. Rev. Lett. 93260601 (2004).

[27] E. Rhoades, E. Gussakovsky, and G. Haran Proceedings of the National Academy of Sciences of the USA 1003197 (2003).

[28] H. Sano, M. Tachiya, J. Chem. Phys. 711276 (1979).

[29] S. N. Majumdar, and A. Comtet Phys. Rev. E 061105 (2002).

[30] V. Balakrishnan, Physica A 132569 (1985)

[31] W. R. Schneider, W. Wyss, J, Math. Phys. 30134 (1989).

[32] A. I. Saichev, and G. M. Zaslavsky Chaos 7753 (1997).

[33] E. Barkai, Chemical Physics 28413 (2002).

[34] R. Gorenflo, A. Vivoli, F. Mainardi, Nonlinear Dynamics 38101 (2004)

[35] E. Barkai, Phys. Rev. E 63046118 (2001).

[36] M. M. Meerschaert, H. P. Scheffler J. of Applied Probability 41623 (2004).

[37] J. Sung and R. J. Silbey Phys. Rev. Lett. 91, 160601 (2003)

[38] K. Seki, M. Wojcik, and M. Tachiya Journal of Chemical Physics 119, 2165 (2003)

[39] A. V. Chechkin, R. Metzler, V. Y. Gonchar, J. Klafter, L. V. Tanatarov J. of Physics A-Mathematical and General 36 L537 (2003).

[40] I. Goychuk, and P. Hanggi Phys. Rev. E 70051915 (2004).

[41] G. R. Kneller, Physical Chemistry Chemical Physics 7 2641 (2005).

[42] W. T. Coffey, J. of Molecular Liquids 1145 (2004).

[43] J. P. Bouchaud J. De Physique I 21705 (1992).

[44] I. G. Sokolov, and R. Metzler Phys. Rev. E 67010101 (2003)

[45] A. Dhar, and S. N. Majumdar Phys. Rev. E 596413 (1999). 